\title{
Phenolic composition, antioxidant, anti-wrinkles and tyrosinase inhibitory activities of cocoa pod extract
}

\author{
Azila Abdul Karim ${ }^{1 \dagger}$, Azrina Azlan $2,3,4^{*}+$, Amin Ismail ${ }^{2}$, Puziah Hashim³ ${ }^{3}$ Siti Salwa Abd Gani ${ }^{3}$, \\ Badrul Hisyam Zainudin ${ }^{1}$ and Nur Azilah Abdullah
}

\begin{abstract}
Background: Cocoa pod is an outer part of cocoa fruits being discarded during cocoa bean processing. Authors found out that data on its usage in literature as cosmetic materials was not recorded in vast. In this study, cocoa pod extract was investigated for its potential as a cosmetic ingredient.

Methods: Cocoa pod extract (CPE) composition was accomplished using UHPLC. The antioxidant capacity were measured using scavenging assay of 1,2-diphenyl-2-picrylhydrazyl (DPPH), $\beta$-carotene bleaching assay (BCB) and ferric reducing antioxidant power (FRAP). Inhibiting effect on skin degradation enzymes was carried out using elastase and collagenase assays. The skin whitening effect of CPE was determined based on mushroom tyrosinase assay and sun screening effect (UV-absorbance at 200-400 nm wavelength).

Results: LC-MS/MS data showed the presence of carboxylic acid, phenolic acid, fatty acid, flavonoids (flavonol and flavones), stilbenoids and terpenoids in CPE. Results for antioxidant activity exhibited that CPE possessed good antioxidant activity, based on the mechanism of the assays compared with ascorbic acid (AA) and standardized pine bark extract (PBE); DPPH: AA > CPE > PBE; FRAP: PBE > CPE > AA; and BCB: BHT > CPE > PBE. Cocoa pod extract showed better action against elastase and collagenase enzymes in comparison with PBE and AA. Higher inhibition towards tyrosinase enzyme was exhibited by CPE than kojic acid and AA, although lower than PBE. CPE induced proliferation when tested on human fibroblast cell at low concentration. CPE also exhibited a potential as UVB sunscreen despite its low performance as a UVA sunscreen agent.
\end{abstract}

Conclusions: Therefore, the CPE has high potential as a cosmetic ingredient due to its anti-wrinkle, skin whitening, and sunscreen effects.

Keywords: Cocoa pod extract, Antioxidant, Anti-wrinkles, Skin whitening, Sunscreen agent, Cosmetic ingredient

\section{Background}

Skin wrinkles form as a result of natural aging process and the presence of excessive amount of reactive oxygen species (ROS) [1]. Although skin has self-defence system to deal with ROS, excessive and chronic exposure to UV can overwhelm the condition leading to oxidative stress and damage resulting premature aging. An imbalance of

\footnotetext{
* Correspondence: azrinaaz@upm.edu.my

${ }^{\dagger}$ Equal contributors

2 Department of Nutrition and Dietetics, Faculty of Medicine and Health Sciences, Universiti Putra Malaysia, 43400 UPM Serdang, Selangor, Malaysia ${ }^{3}$ Laboratory of Halal Science Research, Halal Products Research Institute, Universiti Putra Malaysia, 43400 UPM Serdang, Selangor, Malaysia Full list of author information is available at the end of the article
}

ROS on skin is due to factors such as overexposure to sunlight [2] and lack of essential nutrient intake [3]. In normal condition, skin produces enzymes such elastase and collagenase, at similar rate as aging process occurs and age increases. However, with overexposure to sunlight (UVA and UVB), the presence of excessive ROS and smoking habit [4], the enzymes are produced at a faster rate resulting in faster degradation of elastin and collagen which are the main foundation of extracellular matrix (ECM) of the dermis [5]. Additionally, excessive exposure to sunlight, induced production of melanin in the skin layer. Tyrosinase is the responsible enzyme that initiates skin pigmentation [6]. Potentially, plant extracts 
can suspend the skin aging and pigmentation process [7] by encountering the ROS [3] and protecting the skin from UV [1]. The extracts protect the skin in various ways, including scavenging the ROS, reducing the ROS reactivity, inhibiting the oxidation, absorbing the UV light, suppressing the enzymes, thus reducing the risk of wrinkle formation and protecting the skin from aging. Examples of compounds that have attracted researchers to topical skin applications are polyphenols, triterpenes and stillbenoids. Polyphenols have a phenol ring with at least one hydroxyl substituent that enables scavenging of ROS, reducing metal ions, modulating protein phosphorylation (related to inhibition of enzymes activity), and inhibiting lipid peroxidation [8].

Cocoa pods are agricultural waste abundantly produced in cocoa plantations during the extraction of cocoa beans for cocoa processing. As discarded materials, cocoa pods can initiate the black pod disease by making the ground susceptible to the growth of fungi, which is typical in hot and humid climate. The pods vary in color (from maroon to green) and thickness when ripe depending on their clone. In Malaysia, multi clones of cocoa are planted and when discarded, the pods are thrown away in bulk regardless of the clones. Cocoa pods are used as fertilizers and animal feed. It is also being used as a source of activated carbon (unpublished data), potash, colorant [9], gum [10] and anti hypercholesterolemia supplement [11]. Previously, total phenolic content of cocoa pod husk was determined at $45.6-46.4 \mathrm{mg}$ gallic acid equivalent of soluble phenolic while $32.3 \%$ carbohydrate, $21.44 \%$ lignin, $19.2 \%$ sugars, $8.6 \%$ protein and $27.7 \%$ minerals were reported [12]. Higher level of antioxidant in cocoa pod extract by total phenolic content (TPC) was reported at $49.54 \pm 3.39 \mathrm{mg}$ gallic acid equivalent (GAE) per gram sample and total flavonoid content (TFC) at $22.42+0.98 \mathrm{mg}$ rutin equivalent per gram sample (unpublished data). Sartini et al. [11] also reported higher TPC value of cocoa pod husk at $56.5 \pm$ $0.57 \mathrm{mg} \mathrm{GAE} / \mathrm{g}$. Scientific publication on identification of polyphenol compounds in a cocoa pod is rare, necessitating further research. Therefore, the present experiment was carried out to determine the cocoa pods compounds, which are rich in antioxidant activity and can be turned into high end-value products. In this paper, we investigated the anti-wrinkle, skin whitening and potential UV-protecting properties of cocoa pod extract using invitro assays and identified the potential of this extract as a new active ingredient for cosmetic.

\section{Methods}

\section{Reagents}

Reagents used were of analytical grade purchased from suppliers such as Sigma-Aldrich for 1,2-diphenyl-2picrylhydrazyl (DPPH), L-(+)-ascorbic acid (AA), Linoleic acid, Kojic acid (KA), Tyrosinase (from mushroom) and L-3,4-dihydroxyphenyl-alanine methyl ester hydrochloride (L-DOPA); Merck for acetonitrile, ammonium formate, formic acid, dimethylsulfoxide (DMSO), hydrochloric acid $(\mathrm{HCl})$, chloroform and Tween-20; Fluka for 2,4,6-tripyridyl-s-triazine (TPTZ); Qrec for ferric chloride $\left(\mathrm{FeCl}_{3}\right)$, Friendemann Schmidt for ferrous sulfate (FeS$\left.\mathrm{O}_{4} .7 \mathrm{H}_{2} \mathrm{O}\right), \mathrm{GmbH}$ for ethanol and methanol; and MP Biomedicals for $\beta$-carotene powder. Test kits purchased from Enzo Life Sciences, Inc. Standardized Pine Bark extract (PBE) sample was provided by DKSH Malaysia Sdn Bhd (sample from Horphag, Switzerland) and used as a positive control in addition to ascorbic acid, and butylated hydroxytoluene (BHT, Sigma-Aldrich) in $\beta$-carotene bleaching assay. Human dermal fibroblast, adult (HDFa) was acquired from Life Technologies Corporation (GIBCO, catalogue number C-013-5C; Lot number 1378119) as well as Dulbecco's modified Eagle's medium (DMEM) and Trypsin (Trypsin $\mathrm{LE}^{\mathrm{TM}}$ ). Fetal bovine serum (FBS) and Streptomycin/Penicillin (antibiotics) obtained from Biowest. MTT or 3-(4,5-Dimethylthiazol-2-yl)-2,5-diphenyl tetrazolium bromide supplied by Bio Basic Canada Inc. Phosphate Buffer Saline (PBS, 10X) purchased from R\&M Chemicals, UK.

\section{Sample preparation}

Discarded cocoa pods collected from a cocoa fermentation site at cocoa plantation in Cocoa Development and Research Centre, Jengka, Pahang, Malaysia. After rinsed with tap water, the pods were chopped using mechanical fruit slicer (FC-312, Zhaoqing Fengxiang Food Machinery, China) for better water removal when drying in highperformance dryer (FD-825, Protech, Malaysia). The dried pods were ground into powder at $1.0 \mathrm{~mm}$ size using a grinding machine with a sieve (Automatic Hammer Mill Grinder, China). The cocoa pod powder was kept in tight containers at room temperature (not more than two weeks) until extraction procedure adjourned.

Twenty millilitres of aqueous ethanol (80\%) were poured into a conical flask containing one gram of cocoa pod powder and shaken in water bath shaker (BS-21, Lab Companion, Korea) at $120 \mathrm{rpm}, 40^{\circ} \mathrm{C}$, for 30 minutes to prepare the extract. Soluble portion filtered (using Whattman No.1), evaporated to dryness (using vacuum rotary evaporator; IKA, Germany) and re-dissolved with $5 \mathrm{ml}$ ethanol after recording the weight. Insoluble material was filtered out using filter paper. The extracted sample was stored in air-tight vial at $-10^{\circ} \mathrm{C}$ until evaluated. We used $\mathrm{AA}$ and PBE as the positive control in the study, due to its effective role in maintaining cellular function $[13,14]$ which affects the skin condition.

\section{Identification of CPE compound using LC/MS/MS}

LC-MS/MS analysis was performed using a Perkin Elmer Flexar FX-15 ultra-high performance liquid chromatography 
(Perkin Elmer, USA). It equipped with a reversed-phase C18 analytical column of $50 \mathrm{~mm} \times 2.1 \mathrm{~mm} \times 1.9 \mu \mathrm{m}$ particle size (Perkin Elmer, USA). The column oven temperature was set at $35^{\circ} \mathrm{C}$, and the flow rate was $250 \mu \mathrm{l} / \mathrm{min}$. Mobile phases $\mathrm{A}$ and $\mathrm{B}$ were water and acetonitrile, respectively, each containing $5 \mathrm{mM}$ ammonium formate and $0.1 \%$ formic acid. The linear gradient programme was set as follows: 0-0.1 min, 90\% A/10\% B; $0.1-8 \mathrm{~min}, 10 \% \mathrm{~A} / 90 \% \mathrm{~B}$; $8-10 \mathrm{~min}, 10 \% \mathrm{~A} / 90 \% \mathrm{~B}$; 10-15 $\mathrm{min}, 90 \% \mathrm{~A} / 10 \% \mathrm{~B}$. The injection volume was $20 \mu \mathrm{L}$ with a run time of 15 minutes. The UHPLC was hyphenated to a triple quadrupole mass spectrometer 3200 QTrap (ABSciex) equipped with an electrospray ionization interface set at negative mode. The interface heater held at the temperature of $500^{\circ} \mathrm{C}$ and an ion-spray (IS) voltage of $-4500 \mathrm{eV}$. The nebulising gas (GS1), heating gas (GS2) and curtain gas pressures set at 40, 40 and 10 psi, respectively during the whole analysis. Nitrogen was used as collision and spray gas. Full scan data acquisition was performed, scanning from $\mathrm{m} / \mathrm{z} 5$ to 1500 in enhanced MS IDA EPI mode. Analyst software version 1.5.2 was used for method development, data acquisition and data processing.

\section{Determination of antioxidant activities}

Antioxidant activities were evaluated using three different assays; i.e., 1,2-diphenyl-2-picrylhydrazyl (DPPH), Ferric Reducing Antioxidant Power (FRAP) and $\beta$ carotene bleaching assay (BCB). Methods of preparation of DPPH [2,15,16], FRAP [17-22] and BCB [23] solutions and calculation were adopted with some modifications. Briefly, DPPH solution was prepared by diluting $1.2 \mathrm{ml}$ of DPPH stock solution (0.2 M DPPH in ethanol) with $3 \mathrm{ml}$ ethanol and $0.5 \mathrm{ml}$ DMSO. In a 96-well microplate, $270 \mu \mathrm{l}$ of the solution was added to $30 \mu \mathrm{l}$ of tested sample in a dilution series of $7.8-1000 \mu \mathrm{g} / \mathrm{ml}$, and absorbance measured at $550 \mathrm{~nm}$ [24]. Scavenging effect was calculated by the percentage of faded purple DPPH solution color into yellow by the tested sample against the control (DPPH solution only). The $\mathrm{EC}_{50}$ of $\mathrm{DPPH}$ assay represents the concentration of the tested sample needed to reduce the DPPH by $50 \%$ where the value obtained from linear regression graph.

For FRAP assay, two solutions; i.e., ferric chloride solution ( $3 \mathrm{mM}$ in $5 \mathrm{mM}$ citric acid) and TPTZ solution (2,4,6-tripyridyl-s-triazine; $1 \mathrm{mM}$ in $0.05 \mathrm{M}$ hydrochloric acid) were prepared. Fifteen $\mu \mathrm{l}$ of the tested sample was added to $270 \mu \mathrm{l}$ TPTZ solution and measurement at $620 \mathrm{~nm}$ was carried out immediately after addition of $15 \mu \mathrm{l}$ of ferric chloride solution. The absorbance data calculated against serial dilution of Ferrous sulfate $\left(\mathrm{FeSO}_{4} \cdot 7 \mathrm{H}_{2} \mathrm{O}\right)$ and recorded as equivalent to $\mu \mathrm{M} \mathrm{Fe}{ }^{2+}$. Effective concentration at $50 \%\left(\mathrm{EC}_{50}\right)$ of FRAP value is the sample concentration required to reduce $0.5 \mathrm{~mol}$ of $\mathrm{Fe}^{3+}$ to $\mathrm{Fe}^{2+}$.
Two milligrams of $\beta$-carotene powder was dissolved in $0.2 \mathrm{ml}$ chloroform followed by adding $0.2 \mathrm{ml}$ linoleic acid, $2 \mathrm{ml}$ Tween 20 and $100 \mathrm{ml}$ of distilled water to make $\beta$-carotene solution. It was mixed until almost transparent solution obtained. Two hundred $\mu \mathrm{l}$ of the solution was added to $20 \mu \mathrm{l}$ of the tested solution and measurement at $450 \mathrm{~nm}$ was carried out after incubation for 20 minutes at $50^{\circ} \mathrm{C}$. Measurement was monitored for 2 hours at 30-minute intervals. Calculation of antioxidant activity percentage obtained by the difference of degradation rate of tested sample to the degradation rate of control ( $\beta$-carotene solution only). Effective concentration at $50 \%\left(\mathrm{EC}_{50}\right)$ was determined to represent the ability of the sample to protect the $\beta$-carotene solution from degradation, thus indicating high antioxidant activity with low concentration of the tested sample. Instead of AA, BHT was used as a positive control in this assay. BHT is well-known antioxidant specifically in protecting lipid oxidation [25] and stronger antioxidant compound compared with AA [26].

\section{Determination of elastase and collagenase inhibition}

Elastase and collagenase inhibition measurements were carried out using drug discovery kits (Neutrophil Elastase Colorimetric and MMP-1 Colorimetric, respectively) following protocols as in Enzo Life Science [27,28]. For elastase inhibition assay, $20 \mu \mathrm{l}$ of tested sample was diluted with $65 \mu \mathrm{l}$ buffer-solution containing $100 \mathrm{mM}$ HEPES, $500 \mathrm{mM} \mathrm{NaCl}$ and $0.05 \%$ Tween 20 in DMSO in a 96-well plate. Elastatinal $(100 \mu \mathrm{M})$ used as the control inhibitor. The neutrophil elastase enzyme (purified human neutrophil elastase, $2.2 \mu \mathrm{U} / \mu \mathrm{l}$ ) at $10 \mu \mathrm{l}$ was added to the diluted tested sample and incubated for 10 minutes at $37^{\circ} \mathrm{C}$. Later, $5 \mu \mathrm{l}$ substrate (MeOSucAla-Ala-Pro-Val-pNA, $100 \mu \mathrm{M}$ ) was added to each well and absorbance at $405 \mathrm{~nm}$ was monitored for 10 minutes.

Briefly for collagenase inhibition assay, $20 \mu \mathrm{l}$ of tested sample was diluted with $50 \mu \mathrm{l}$ buffer-solution $(50 \mathrm{mM}$ HEPES, $10 \mathrm{mM} \mathrm{CaCl}_{2}, 0.05 \%$ Brij-35 and $1 \mathrm{mM}$ DTNB in DMSO). Twenty microlitres of MMP-1 enzyme (E.coli recombinant human MMP-1 catalytic domain, $153 \mathrm{mU} /$ $\mu \mathrm{l})$ was added to each well prior to incubation at $37^{\circ} \mathrm{C}$ for 30 minutes. Control inhibitor, NNGH (N-Isobutyl-N(4-methoxyphenylsulfonyl) glycylhydroxamic acid; $1.3 \mu \mathrm{M}$ ), was used for comparison. Substrate (thiopeptide, Ac-PLG[2-mercapto-4-methyl-pentanoyl]-LG-OC $\left.\mathrm{O}_{2} ; 100 \mu \mathrm{M}\right)$ at $10 \mu \mathrm{l}$ was added to each well and absorbance at $410 \mathrm{~nm}$ was monitored for 10 minutes.

For both protocols, slope of remaining activity for the tested sample against the control (without sample) was calculated in percentage and inhibition percentage was obtained by subtracting the obtained value from 100 . Inhibition concentration of $50 \%\left(\mathrm{IC}_{50}\right)$ is the concentration 
Table 1 Product ion of cocoa pod extract in negative mode

\begin{tabular}{|c|c|c|c|c|c|}
\hline Class & Compound & $\begin{array}{l}\text { Retention time, } \\
t_{R}(\min )\end{array}$ & $m / z$ & Reference & in $P B E$ \\
\hline Sugar & $\begin{array}{l}\text { Gluconic acid sodium } \\
\text { salt/glucose acid }\end{array}$ & 0.787 & $195,177,129,85,75$ & 34,35 & Yes \\
\hline Carboxylic acid & Tartaric acid & 1.184 & $149,105,87,73$ & 34 & No \\
\hline Flavonoid/flavonol & Rhamnetin & 1.315 & $315,241,139,108,97,80,70$ & 34 & No \\
\hline Carboxylic acid & Citric acid & 1.447 & $191,111,87$ & 53,35 & Yes \\
\hline Phenolic acid & Protocatechuic acid & 1.842 & $153,109,65$ & 38,39 & Yes \\
\hline Phenolic acid & $\begin{array}{l}\text { Protocathecuic } \\
\text { derivatives/nucleotide }\end{array}$ & 1.972 & $329,241,199,108,97,69$ & $41 / 35$ & No \\
\hline Phenolic acid & $\begin{array}{l}\text { Protocathecuic } \\
\text { derivatives/nucleotide }\end{array}$ & 1.974 & $329,241,139,97,80$ & $41 / 35$ & No \\
\hline Unknown & & 2.106 & $330,241,122,96,80$ & - & No \\
\hline Phenolic acid & p-hydroxybenzoic acid & 2.238 & 137,93 & 36,40 & Yes \\
\hline Phenolic acid & Salicyclic acid & 2.238 & $137,108,91,65$ & 35 & Yes \\
\hline Unknown & & 2.500 & $363,241,122,96,59$ & - & No \\
\hline Phenolic acid & Methyl salicylate & 2.633 & $151,139,124,91,65$ & 35 & No \\
\hline Nucleotide & disodium salt, nucleotide & 2.763 & $401,327,69$ & 35 & No \\
\hline Phospholipid & Phospholipid derivatives & 2.897 & $381,249,161,113,98,85,68$ & 35 & No \\
\hline Glucosinolate & Glucosinolate derivatives & 3.029 & $379,155,59$ & 35 & No \\
\hline Flavonoid & Sineginhomoorientin derivatives & 3.161 & $355,225,207,96,69,59$ & 35,42 & No \\
\hline Unknown & & 3.292 & $358,245,222,178,161,135,123$ & - & No \\
\hline Unknown & & 3.293 & $358,222,178,161,151,135,123$ & - & Yes \\
\hline Unknown & & 3.423 & $452,408,372,328,285,250,230,190,178,160,148,135$ & - & No \\
\hline Flavones-Sugar & Apigenin-c-glucose-c-pentoside & 3.425 & $563,545,473,443,395,383,365,353,325,311,297,233$ & 43 & No \\
\hline Unknown & & 3.557 & $357,241,139,96$ & - & No \\
\hline Glucosinolate & Glucosinolate derivatives & 3.688 & $372.2,285.1,136,178,147,160.2,135$ & 35 & No \\
\hline Glucosinolate & Glucosinolate derivatives & 3.822 & $439.2,314,300.1,269.1,180.1,151.1,96.9,80.1$ & 35,42 & No \\
\hline Glucosinolate & Glucosinolate derivatives & 4.086 & $371,281,241,151,96$ & 35 & No \\
\hline Terpenoid & Terpenoid derivatives & 4.351 & $345,201,171,155,59$ & 35 & No \\
\hline Flavonoid/Flavonol & Kaempferol derivatives & 4.879 & $723,677,659,550,451,367,225$ & 34 & Yes \\
\hline Terpenoid & Terpenoid derivatives & 5.010 & $345,99,59$ & 35 & No \\
\hline Flavonoid & Flavone derivatives & 5.143 & $327,309,197$ & 35 & No \\
\hline Unknown & & 5.404 & $329,229,211,171,139,99$ & - & No \\
\hline Flavone & Flavone/luteolin & 5.407 & $329,311,229,211,171,139,127,99,69$ & 44 & No \\
\hline Flavonoid & Flavone derivatives & 5.535 & $327,205,183,171,69,59$ & 35 & No \\
\hline Ketone-Sugar & Ribulose1,5-bisphosphate & 5.667 & $309,265,209,193,151,137,109$ & 53 & No \\
\hline Terpenoid & Crysoplenol & 5.802 & 330,172 & 35 & No \\
\hline Flavonoid & Flavones derivatives & 6.199 & $305,287,249,163,135,93$ & $37,54,45,46$ & No \\
\hline Flavones-Sugar & apigenin glycoside & 6.594 & $313,295,183,171,129,99,58$ & 34,44 & No \\
\hline Phenylpropanoid & Chlorogenic acid derivatives & 6.858 & $353,97,80$ & 34 & No \\
\hline Unknown & & 7.377 & $339,239,183,170,99$ & - & Yes \\
\hline Flavonoid & Flavones derivatives & 7.384 & $295,277,181,171$ & 35 & No \\
\hline Flavonoid & Flavones derivatives & 7.516 & $293,249,195,113$ & 35 & No \\
\hline Flavonoid & Flavones derivatives & 7.516 & $293,249,236,220,205,190,177,164,148,81$ & 35 & No \\
\hline Carboxylic acid & Malic acid & 8.042 & $133,115,71$ & $34,47,48,49,53,35$ & Yes \\
\hline
\end{tabular}


Table 1 Product ion of cocoa pod extract in negative mode (Continued)

\begin{tabular}{|c|c|c|c|c|c|}
\hline Stillbenoids & Resveratrol & 8.567 & 277 & 50 & No \\
\hline Stillbenoids & Resveratrol & 8.567 & 227 & 37,51 & No \\
\hline Fatty acid & Linoleic acid & 9.094 & 279 & 41 & No \\
\hline Vitamin & Flavin mononucleotide & 9.218 & 455 & 43 & No \\
\hline Fatty acid & Oleic acid & 9.620 & 281 & 41 & No \\
\hline Flavonoid/flavone & Linarin/acacetin & 14.350 & 283 & 52 & Yes \\
\hline
\end{tabular}

of the tested sample that can inhibit the enzymes activities to $50 \%$.

\section{Determination of skin whitening and potential UV-sunscreen activities}

Skin whitening effect was evaluated based on inhibition of mushroom tyrosinase by the tested sample with L-DOPA as substrate using a method described by Chiari et al. [29]. The tested solution was diluted in series $(1000-250 \mu \mathrm{g} / \mathrm{ml})$ using DMSO and $20 \mu \mathrm{l}$ was pipetted into a 96-well microplate, followed by addition of $138 \mu \mathrm{l}$ PBS (phosphate buffer solution) and $2 \mu \mathrm{l}$ mushroom tyrosine solution $\left(2500 \mathrm{U} / \mathrm{ml}\right.$, in PBS). After incubation at $37^{\circ} \mathrm{C}$ for $90 \mathrm{mi}-$ nutes, $40 \mu \mathrm{l}$ of L-DOPA (2.5 mM in PBS) was added, and measurement at $450 \mathrm{~nm}$ monitored for 20 minutes. Kojic acid was used for comparison.

For UV sunscreen potential activity, the tested sample was dissolved in ethanol (ratio 8:125). Same solvent was used as blank for baseline correction so that the results obtained could be compared with each other. Samples were scanned at 200-400 nm wavelength using dual beam UVSpectrophotometer (Cary 60, US). Absorbance of tested samples at critical wavelengths (290, 308, 330 and $350 \mathrm{~nm}$ ) [30] were selected for comparison. Higher value of absorbance indicates better potential as UV-sunscreen agent, invitro. Two broad spectrums of commercial sunscreens; i.e. Avobenzone and Octylmethoxycinnamate (OMC) were used for comparison. The results obtained were only for screening purposes; therefore, a subsequent experimental study using non-invasive method had to be carried out to determine the Sun Protecting Factor (SPF) value prior to using the extracts in formulation.

\section{Cell viability using human dermal fibroblast}

Healthy cells were initiated from cryopreserved HDFa in a $25 \mathrm{~cm}^{2}$ tissue culture flask in DMEM containing $10 \%$

Table 2 Extra compound of pine bark in negative mode

\begin{tabular}{|c|c|c|c|c|}
\hline Class & Compound & Retention time, $t_{R}(\min )$ & $m / z$ & Reference \\
\hline Unknown & & 1.314 & $399,353,221,207,161,85$ & - \\
\hline Phenolic acid & Gallic acid & 1.446 & $169,125,95,79,67$ & 35 \\
\hline Phenolic acid & Methyl benzoate acid/ caffeic acid & 1.841 & $135,108,93,80$ & $34,55,56,57$ \\
\hline Phenolic acid & 3,5-dihydroxybenzoic acid & 1.841 & $109,91,65$ & 35 \\
\hline Unknown & & 2.369 & 445,137 & - \\
\hline Unknown & & 2.634 & $459,137,93$ & - \\
\hline Unknown & & 3.564 & $466,438,303,285,275,231,175,151,125,82,57$ & - \\
\hline Flavonoid/flavanol & Quercetin derivatives & 4.229 & $506,459,340,151,165,125$ & 35 \\
\hline Unknown & & 4.494 & $350,290,244,135$ & - \\
\hline Unknown & & 5.159 & $291,247,217$ & - \\
\hline Unknown & & 5.424 & $365,321,247,227,165,151$ & - \\
\hline Flavonoid/flavanol & Catechin & 5.424 & $289,173,162,137,122,109$ & 35 \\
\hline Unknown & & 5.557 & $349,305,287,269,207,189,177,161,147,85$ & - \\
\hline Unknown & & 5.689 & $329,255,227$ & - \\
\hline Carboxylic acid & Gibbrellin derivatives & 5.821 & $331,287,269,253,244,161$ & 34 \\
\hline Unknown & & 6.085 & 333,273 & - \\
\hline Flavonoid & Flavonol derivatives & 7.008 & 315,269 & 35 \\
\hline Fatty acid & Decanoic acid & 8.325 & 171,127 & - \\
\hline Phenolic acid & Caffeic acid & 13.980 & $179,161,135,109,89$ & 35 \\
\hline
\end{tabular}


Table 3 Antioxidant activities of CPE, AA and PBE based on (a) Scavenging effect using DPPH assay (b) Metal reducing ion capability, and (c) Antioxidant activity using $\beta$-carotene bleaching assay

\begin{tabular}{|c|c|c|c|c|c|c|c|c|c|c|}
\hline \multirow[t]{2}{*}{ Assay } & \multirow[t]{2}{*}{ Sample } & \multicolumn{8}{|c|}{ Concentration, $\mu \mathrm{g} / \mathrm{ml}$} & \multirow[t]{2}{*}{$E C_{50}$} \\
\hline & & 7.8 & 15.6 & 31.3 & 62.5 & 125 & 250 & 500 & 1000 & \\
\hline \multirow[t]{3}{*}{ (a) DPPH assay (\%) } & AA & $45.03 \pm 1.74^{\mathrm{aa}}$ & $50.14 \pm 3.20^{\mathrm{a} \beta}$ & $63.05 \pm 6.39^{\mathrm{a \gamma}}$ & $66.63 \pm 1.75^{\text {by }}$ & $72.21 \pm 2.21^{\mathrm{b \delta}}$ & $73.45 \pm 2.24^{\mathrm{b} \delta}$ & $76.48 \pm 2.58^{\mathrm{b} \delta}$ & $77.53 \pm 2.67^{\mathrm{b} \delta}$ & 13.25 \\
\hline & PBE & $13.70 \pm 0.27^{\mathrm{ba}}$ & $23.92 \pm 1.04^{\complement \beta}$ & $38.49 \pm 2.26^{\text {by }}$ & $58.89 \pm 3.16^{\complement \delta}$ & $67.43 \pm 1.71^{\complement \varepsilon}$ & $69.93 \pm 1.91^{\mathrm{b} \varepsilon}$ & $73.54 \pm 1.85^{\mathrm{b} \varepsilon}$ & $74.86 \pm 1.42^{\mathrm{be}}$ & 49.33 \\
\hline & CPE & $10.80 \pm 0.63^{c a}$ & $32.10 \pm 0.40^{\mathrm{b} \beta}$ & $58.98 \pm 5.82^{\mathrm{a \gamma}}$ & $87.07 \pm 3.09^{\mathrm{a} \delta}$ & $85.01 \pm 4.44^{\mathrm{a} \delta}$ & $86.81 \pm 3.47^{\mathrm{a} \delta}$ & $84.52 \pm 3.23^{\mathrm{a} \delta}$ & $83.28 \pm 0.66^{\mathrm{a} \delta}$ & 26.10 \\
\hline \multirow[t]{3}{*}{ (b) FRAP assay $\left(\mu \mathrm{M} \mathrm{Fe}^{2+}\right)$} & AA & $416.24 \pm 78.22^{\mathrm{aba} \beta}$ & $610.43 \pm 3.99^{\text {aү } \delta}$ & $405.47 \pm 42.79^{\mathrm{ba} \beta}$ & $454.19 \pm 31.55^{c \beta}$ & $336.24 \pm 45.33^{c a}$ & $534.53 \pm 29.57^{\text {by }}$ & $599.83 \pm 100.81^{\text {by }}$ & $754.02 \pm 72.49^{\text {aүع }}$ & 204.25 \\
\hline & PBE & $398.11 \pm 15.95^{b a}$ & $485.06 \pm 39.38^{\mathrm{b} \beta \gamma}$ & $483.67 \pm 53.55^{\mathrm{a} \beta \gamma}$ & $509.22 \pm 46.27^{\text {by }}$ & $551.44 \pm 45.71^{\text {aү } \delta}$ & $435.61 \pm 57.16^{c \beta \gamma}$ & $793.94 \pm 41.84^{\mathrm{a} \varepsilon}$ & $587.83 \pm 4.71^{\mathrm{b} \delta}$ & 70.76 \\
\hline & CPE & $478.12 \pm 46.06^{\mathrm{a} \beta}$ & $567.86 \pm 38.07^{\mathrm{a \gamma}}$ & $390.94 \pm 15.23^{b a}$ & $684.27 \pm 18.72^{\mathrm{a} a \varepsilon}$ & $526.66 \pm 42.06^{\mathrm{a} \beta \gamma}$ & $620.68 \pm 9.07^{\mathrm{a} \delta}$ & $638.80 \pm 52.31^{\mathrm{b} \delta}$ & $729.57 \pm 57.20^{\mathrm{a} \varepsilon}$ & 97.30 \\
\hline \multirow[t]{3}{*}{ (c) BCB assay (\%) } & BHT & $31.01 \pm 3.60^{\mathrm{aa}}$ & $46.83 \pm 3.18^{\mathrm{a} \beta}$ & $59.26 \pm 1.46^{\mathrm{a \gamma}}$ & $78.70 \pm 3.16^{\mathrm{a} \delta}$ & $91.72 \pm 2.51^{\mathrm{a} \varepsilon}$ & $98.23 \pm 6.09^{a \varepsilon}$ & $97.17 \pm 2.82^{\mathrm{a} \varepsilon}$ & $99.17 \pm 5.48^{\mathrm{a} \varepsilon}$ & 24.09 \\
\hline & PBE & $3.67 \pm 0.27^{\mathrm{ca}}$ & $12.88 \pm 2.26^{\mathrm{b} \beta}$ & $23.20 \pm 7.65^{\text {by }}$ & $25.31 \pm 6.89^{c \gamma}$ & $40.71 \pm 8.66^{\mathrm{c \gamma \delta}}$ & $48.32 \pm 7.73^{c \delta}$ & $49.59 \pm 3.51^{c \delta}$ & $63.68 \pm 13.15^{c \delta}$ & 302.71 \\
\hline & CPE & $9.65 \pm 1.99^{\mathrm{b} \beta}$ & $5.15 \pm 1.10^{c a}$ & $5.74 \pm 1.48^{\mathrm{ca}}$ & $51.47 \pm 5.64^{\text {by }}$ & $70.50 \pm 0.43^{\mathrm{b} \delta}$ & $81.55 \pm 1.08^{b \varepsilon}$ & $83.44 \pm 0.80^{\mathrm{bf}}$ & $88.08 \pm 2.73^{b €}$ & 84.67 \\
\hline
\end{tabular}

a b c different alphabet in column means significantly different within sample for each assay.

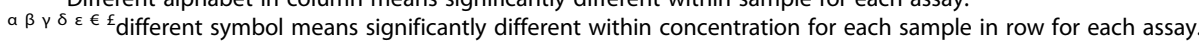


Table 4 Elastase inhibition activity with elastatinal at $100 \mu \mathrm{M}$ for positive control inhibitor for CPE, AA and PBE

\begin{tabular}{|c|c|c|c|c|}
\hline \multirow[t]{2}{*}{ Sample } & \multicolumn{3}{|c|}{ Concentration $(\mu \mathrm{g} / \mathrm{ml})$} & \multirow[t]{2}{*}{$I C_{50}$} \\
\hline & 10 & 100 & 500 & \\
\hline Elastatinal, $100 \mu \mathrm{M}$ & & $30.94 \pm 1.47^{b}$ & & - \\
\hline AA & $36.02 \pm 1.83^{\mathrm{a} \beta}$ & $27.51 \pm 2.59^{\mathrm{ba}}$ & - & 232.29 \\
\hline PBE & $8.57 \pm 0.63^{\mathrm{da}}$ & $34.96 \pm 1.47^{\mathrm{a} \beta}$ & $43.28 \pm 0.05^{\mathrm{ay}}$ & 31.93 \\
\hline$C P E$ & $15.57 \pm 0.86^{\subset \beta}$ & $23.47 \pm 2.15^{\text {by }}$ & $2.54 \pm 0.04^{c a}$ & 3.51 \\
\hline
\end{tabular}

a b c d Different alphabet in column means significantly different within sample.

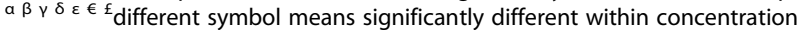
for each sample in row.

FBS and 1\% antibiotics. The cells were incubated at $37^{\circ} \mathrm{C}, 95 \%$ humidity and $5 \% \mathrm{CO}_{2}$ until confluent. The growth media was refreshed every two days until at least $80 \%$ confluence was achieved, and was trypsinized with Trypsin $L^{\mathrm{TM}}$ to passage for not more than eight times for cell viability study [31]. The cells were seeded into a 96-well microplate at density of $1 \times 10^{5}$ per well and incubated for 24 hours. Serial dilution of CPE, AA and $\mathrm{PBE}$ were added to the well, respectively, after removal of the spent media and incubated for another 24 hour. Forty microliters $(40 \mu \mathrm{L})$ of MTT in PBS $(2.5 \mathrm{mg} / \mathrm{mL})$ was added to each well and incubated for 4 hours before the absorbance was measured at 570 with $630 \mathrm{~nm}$ as reference wavelength. A hundred microlitres $(100 \mu \mathrm{L})$ of DMSO was used to dissolve the dye crystals [32]. The percentage of cell viability was calculated based on the optical density of each well against the control (cell without any treatment). Inhibition concentration of $90 \%\left(\mathrm{IC}_{90}\right)$ is the sample concentration that enables $90 \%$ of cells survived after treatment with the tested sample which metabolized the MTT salts to formazan [33].

\section{Statistical analysis}

The results were presented as mean \pm standard deviation determined in triplicates of two independent samples. Comparison was made using two samples $T$-test by Minitab Software version 14.12.0 (US Inc.). Results were significantly different when $\mathrm{p}$-value was less than 0.05 $(\mathrm{p}<0.05)$.

\section{Results and discussion}

Identification of CPE compound using LC/MS/MS

Crude CPE was analyzed by LC/MS/MS in negative mode. We identified each compound based on the literature search in established spectrum databases [34,35]. Product ion spectrum of CPE was compared with the product ion spectrum obtained from the search and was listed in Table 1 [34-54] according to its retention time. We considered the compound as its derivatives, if the product ion spectrum did not hit any of the product ion $\mathrm{m} / \mathrm{z}$ in the search but hit only the first and one or more of the product ion $\mathrm{m} / \mathrm{z}$ in the spectrum. PBE, known to contain a wide range of polyphenol compounds [36], was also analyzed for comparison purposes. Compounds that are similarly available in PBE also were noted at the end column of Table 1. Additionally, compounds that appear only in PBE were listed in Table 2 [34,35,55-57]. $\mathrm{CPE}$ and PBE contained almost similar compounds, including polyphenols, carboxylic acid (citric acid and malic acid) and sugars (gluconic acid sodium salt). Polyphenolic compounds in CPE included phenolic acids (protocatechuic acid, p-hydroxybenzoic acid and salicyclic acid), flavonols (kaempferol) and flavones (linarin), which also were detected in PBE. Resveratrol, a stilbenoids compound that was detected in wine [37], was also found in CPE but not in PBE. CPE also was detected with tartaric acid, apigenin and luteoin (flavone), crysoplenol (terpenoids), linoleic acid and oleic acid (fatty acids) which were not available in PBE. Nevertheless, gallic acid, catechin [58], quercetin and caffeic acid were found in PBE but were absent in CPE.

\section{Antioxidant activities}

Absorbance at $550 \mathrm{~nm}$ of cocoa pod extract was measured after at least 10 minutes of incubation at room temperature by DPPH assay to ensure complete reaction. The incubation time was determined previously (data not included) by a method as described by Marxen et al. [24]. CPE achieved optimum value of scavenging percentage by this assay at $62.5 \mu \mathrm{g} / \mathrm{ml}$, which was lower than PBE and AA at $125 \mu \mathrm{g} / \mathrm{ml}$ (Table 3). At this optimum value, CPE showed significantly higher antioxidant activity $(87.07 \pm 3.09 \% ; 62.5 \mu \mathrm{g} / \mathrm{ml})$

Table 5 Collagenase inhibition activity of CPE, AA and PBE with NNGH at $1.3 \mu \mathrm{M}$ for positive control inhibitor

\begin{tabular}{|c|c|c|c|c|c|c|c|c|c|}
\hline \multirow[t]{2}{*}{ Sample } & \multicolumn{8}{|c|}{ Concentration $(\mu \mathrm{g} / \mathrm{ml})$} & \multirow[t]{2}{*}{$I C_{50}$} \\
\hline & 7.8 & 15.6 & 31.3 & 62.5 & 125 & 250 & 500 & 1000 & \\
\hline $\mathrm{NNGH}, 1.3 \mu \mathrm{M}$ & \multicolumn{8}{|c|}{$95.91 \pm 0.23^{a}$} & \\
\hline AA & $31.87 \pm 3.40^{c a}$ & $44.06 \pm 0.07^{\subset \beta}$ & $48.70 \pm 2.92^{C \gamma}$ & $33.84 \pm 1.00^{d a}$ & $31.70 \pm 2.36^{\mathrm{ca}}$ & $61.75 \pm 8.51^{\mathrm{bc} \delta}$ & $52.91 \pm 4.24^{\mathrm{ay}}$ & $90.16 \pm 1.27^{a \varepsilon}$ & 261.38 \\
\hline PBE & $54.96 \pm 6.77^{\mathrm{b} \delta \varepsilon \epsilon}$ & $50.81 \pm 0.90^{\mathrm{b} \delta}$ & $53.32 \pm 0.53^{\mathrm{b} \varepsilon}$ & $54.04 \pm 1.48^{\mathrm{b} \varepsilon}$ & $16.79 \pm 2.27^{\mathrm{d} a}$ & $60.56 \pm 4.98^{\mathrm{c \epsilon}}$ & $46.00 \pm 1.64^{\text {by }}$ & $39.26 \pm 3.98^{c \beta}$ & 356.01 \\
\hline CPE & $53.15 \pm 0.95^{\mathrm{b} \delta}$ & $48.81 \pm 2.55^{\text {by }}$ & $53.23 \pm 0.41^{b \delta}$ & $50.71 \pm 0.31^{\mathrm{c \gamma}}$ & $38.53 \pm 4.22^{\mathrm{b} \beta}$ & $68.71 \pm 2.12^{\mathrm{b} \varepsilon}$ & $29.97 \pm 1.36^{\mathrm{ca}}$ & $71.44 \pm 2.28^{\mathrm{bc}}$ & 111.29 \\
\hline
\end{tabular}

a b c d Different alphabet in column means significantly different within sample.

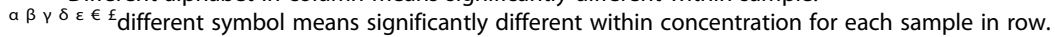


Table 6 Skin whitening activity (\%) of CPE, AA and PBE using mushroom tyrosinase against kojic acid (KA)

\begin{tabular}{|c|c|c|c|c|c|c|c|c|c|}
\hline \multirow[t]{2}{*}{ Sample } & \multicolumn{8}{|c|}{ Concentration, $\mu \mathrm{g} / \mathrm{ml}$} & \multirow[t]{2}{*}{$I C_{50}$} \\
\hline & 7.8 & 15.6 & 31.3 & 62.5 & 125 & 250 & 500 & 1000 & \\
\hline KA & $18.07 \pm 0.25^{\mathrm{b} \beta}$ & $22.44 \pm 0.04^{\text {by }}$ & $11.17 \pm 1.71^{\mathrm{ca}}$ & $11.71 \pm 1.27^{c a}$ & $24.12 \pm 4.89^{\text {by }}$ & $52.22 \pm 7.46^{\mathrm{b} \delta}$ & $47.78 \pm 4.07^{b \delta}$ & $68.80 \pm 2.89^{\complement \varepsilon}$ & 572.28 \\
\hline AA & $8.29 \pm 0.81^{c \beta}$ & $7.25 \pm 0.08^{c a}$ & $14.15 \pm 2.88^{\text {by } \delta \varepsilon}$ & $13.51 \pm 1.40^{c} \delta$ & $11.84 \pm 0.47^{C \gamma}$ & $25.26 \pm 0.89^{d}$ & $18.61 \pm 2.71^{c \varepsilon}$ & $29.62 \pm 1.51^{d \epsilon}$ & 670.82 \\
\hline PBE & $42.57 \pm 1.98^{\mathrm{a} \beta \gamma}$ & $41.22 \pm 2.21^{\mathrm{a} \beta}$ & $43.14 \pm 2.78^{\mathrm{a} \beta \gamma}$ & $44.16 \pm 0.79^{a \gamma}$ & $48.31 \pm 2.71^{\mathrm{a} \delta}$ & $36.67 \pm 0.40^{c a}$ & $49.10 \pm 2.97^{b \delta}$ & $77.65 \pm 4.51^{b \varepsilon}$ & 315.16 \\
\hline CPE & $16.17 \pm 2.67^{b a}$ & $21.52 \pm 2.29^{\mathrm{b} \beta}$ & $16.97 \pm 3.48^{\mathrm{ba} \beta}$ & $27.78 \pm 3.19^{\text {by }}$ & $41.99 \pm 6.93^{\mathrm{a} \delta}$ & $62.23 \pm 0.47^{a \varepsilon}$ & $65.87 \pm 4.20^{\mathrm{a} \varepsilon}$ & $86.51 \pm 2.12^{\mathrm{a}} €$ & 357.95 \\
\hline
\end{tabular}

a b c d Different alphabet in column means significantly different within concentration.

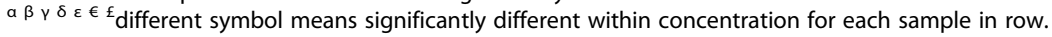

compared with AA $(72.21 \pm 2.21 \% ; 125 \mu \mathrm{g} / \mathrm{ml})$ and PBE $(67.43 \pm 1.71 \% ; 125 \mu \mathrm{g} / \mathrm{ml})$. The effective concentration to reduce the $\mathrm{DPPH}$ radical to $50 \%\left(\mathrm{EC}_{50}\right)$ was determined by plotting linear regression curve of DPPH activity versus ratio of sample concentration to DPPH as previously reported [24]. The tested sample with $\mathrm{EC}_{50}$ value lower than $30 \mu \mathrm{g} / \mathrm{ml}$ by DPPH assay, has high efficiency as free radical scavenger [2], specifically against superoxide anion radicals [59], as exhibited by CPE, which was two times higher antioxidant activity than standardized PBE, although it indicated a two-fold decrease in comparison with AA. The presence of more than one carboxylic acid such as citric acid and malic acid, besides phenolic acid and other polyphenols in $\mathrm{CPE}$ is suggested to contribute to high antioxidant value by DPPH assay [59], compared with AA, which contained a single compound. In addition, higher antioxidant activity values by DPPH assay for CPE than $\mathrm{PBE}$ is also attributed to the presence of terpenoid and resveratrol compounds.

The ability of the tested solution to deviate the mechanism of Fenton reaction by chelating the metal ions [60], such as $\mathrm{Fe}^{2+}$ and $\mathrm{Cu}^{2+}$, which is responsible to convert the hydrogen peroxide to hydroxyl radical (ROS) on the skin [6], can be measured using FRAP assay. We conducted a kinetic study for the tested sample at $620 \mathrm{~nm}$ (data not published) measured after five minutes of reaction. CPE had similar antioxidant activity to AA when measured using FRAP assay except at $250 \mu \mathrm{g} / \mathrm{ml}$, where the FRAP value of CPE was significantly higher than $\mathrm{AA}$ and PBE. Antioxidant activity of PBE was proportional to the tested concentration and showed significantly higher activity when compared with $\mathrm{EC}_{50}$ value of the FRAP assay. Although CPE had three times higher metal reducing ion potential than $\mathrm{AA}$, its performance was 1.4 times lower than that of PBE. The presence of chlorogenic acid derivatives in CPE is likely to contribute to its higher FRAP value than that of AA since this compound is metal chelating ion [60]. Other than that, flavonol derivatives are also known to chelate metal ions [25], such as rhamnetin and kaempferol derivatives in CPE. Flavanol compounds, such as catechin and quercetin also contribute to the chelating action on metal ion [26], which are available in PBE but absent in CPE, resulting in higher FRAP value for PBE. Phenolics with ion reducing ability diminish the possibility of hydroxyl radical's formation path from superoxide anion radicals and additionally inhibit enzymes due to their abilities to chelate copper at the active site [6].

$\beta$-carotene in $\mathrm{BCB}$ assay serves as an indicator that degrades during the oxidation process when linoleic acid turns to hydroperoxides at high incubation temperature [61]. The presence of antioxidant eliminates or reduces the action of this radical species to $\beta$-carotene as measured by the absorbance at $450 \mathrm{~nm}$ and calculation of degradation rate. High value of antioxidant activity by $\mathrm{BCB}$ assay exhibits the tested solution is a good antioxidant agent due to the presence of linoleic acid that acts as pro-oxidant [21]. CPE achieved optimum value of antioxidant activity at concentration of $250 \mu \mathrm{g} / \mathrm{ml}$ $(81.55 \pm 1.08 \%)$, which was higher than standardized PBE and BHT at $125 \mu \mathrm{g} / \mathrm{ml}(40.71 \pm 8.66 \%$ and $91.72 \pm 2.51 \%$,

Table 7 Absorbance of CPE at critical wavelength in comparison with commercial sunscreen agents and standardized pine bark extract

\begin{tabular}{ccccc}
\hline Sample & \multicolumn{3}{c}{ Wavelength (nm) } \\
\cline { 2 - 5 } & $\mathbf{2 9 0}(\mathbf{U V B})$ & $\mathbf{3 0 8}$ (UVB) & $\mathbf{3 3 0}$ (UVA) & $\mathbf{3 5 0}$ (UVA) \\
\hline Avobenzone & $2.080 \pm 0.033^{\mathrm{c}}$ & $1.926 \pm 0.052^{\mathrm{b}}$ & $2.519 \pm 0.091^{\mathrm{a}}$ & $2.723 \pm 0.247^{\mathrm{a}}$ \\
OMC & $2.145 \pm 0.002^{\mathrm{b}}$ & $2.126 \pm 0.030^{\mathrm{a}}$ & $2.413 \pm 0.124^{\mathrm{a}}$ & $2.796 \pm 0.145^{\mathrm{a}}$ \\
PBE & $0.589 \pm 0.009^{\mathrm{d}}$ & $0.231 \pm 0.005^{\mathrm{c}}$ & $0.126 \pm 0.003^{\mathrm{c}}$ & $0.063 \pm 0.002^{\mathrm{c}}$ \\
CPE & $2.639 \pm 0.010^{\mathrm{a}}$ & $2.130 \pm 0.120^{\mathrm{a}}$ & $1.566 \pm 0.200^{\mathrm{b}}$ & $1.007 \pm 0.150^{\mathrm{b}}$ \\
\hline
\end{tabular}

a b c dDifferent alphabet in column means significantly different within sample at each wavelength. 


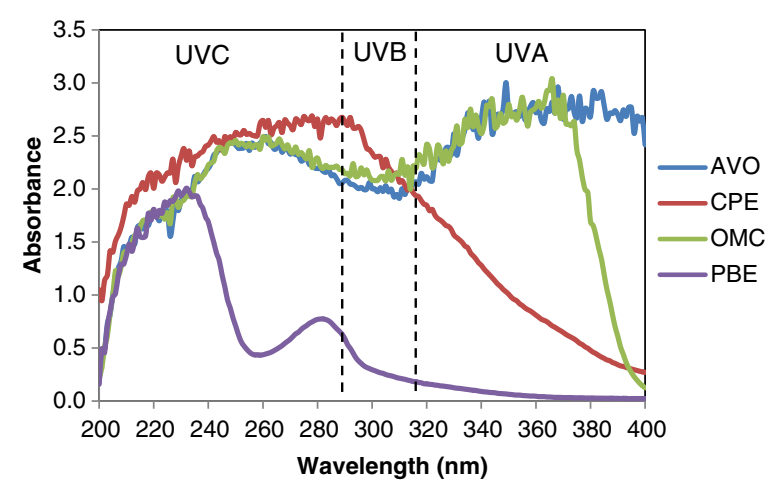

Figure 1 Absorbance of tested samples compared with commercial UV-sunscreen agent (Avobenzone and Octylmethoxycinnamate) at 200-400 wavelength.

respectively). Both $\mathrm{CPE}$ and $\mathrm{PBE}$ had lower antioxidant activity than $\mathrm{BHT}$, but based on $\mathrm{EC}_{50}$ value; antioxidant activity of CPE was four times lower than that of BHT while PBE showed the lowest activity. Higher antioxidant activity of CPE in comparison with PBE can be attributed to the terpenoid and resveratrol compounds that solely indicate better protection for lipid oxidation [62,63]. Plant extracts with lipid protection properties can protect the skin from lipid peroxyl radicals that attack stratum corneum layer [63].

\section{Elastase and collagenase inhibition}

Determination of elastase inhibition at higher concentration $(1000 \mu \mathrm{g} / \mathrm{ml})$ of CPE and standardized PBE resulted in a negative slope, which was also observed for $500 \mu \mathrm{g} /$ $\mathrm{ml}$ of AA, indicating no activity of elastase either for inhibition or activation. This situation should be taken into consideration to avoid false positive results, where the inhibition detects at certain concentration range of extracts or control inhibitors; i.e. elastatinal $(100 \mu \mathrm{M})$ as in Enzo Life Science Manual [27] as well as the concentration of enzyme (neutrophil elastase enzyme, $100 \mu \mathrm{M}$ ) used in this study. Table 4 summarizes the result of elastase inhibition for CPE and standardized PBE at concentrations of 500, 100 and $10 \mu \mathrm{g} / \mathrm{ml}$. At high concentrations (500 and $100 \mu \mathrm{g} / \mathrm{ml}$ ), standardized PBE exhibited higher activity than control inhibitor, which was in agreement with a previous report by Grimm et al. [64]. Catechin, a flavanol was reported to have an inhibitory effect on elastase enzyme $[65,66]$, which is also present in PBE. Catechin was also reported to inhibit binding activity of transcription factors and kinases (AP1 and NF- $\mathrm{kB}$ ), therefore lowering the activity of MMPs for collagen degradation and inflammation [67]. CPE has lower elastase inhibition activity in comparison with standardized PBE at $100 \mu \mathrm{g} / \mathrm{ml}$ but similar to AA and control inhibitor. Terpenoid compounds were previously suggested as elastase inhibitors [65], is present in CPE since flavones and flavonol components are not efficient inhibitors of elastase activity [66]. The inhibition of elastase by CPE was much lower at lower concentration $(10 \mu \mathrm{g} / \mathrm{ml})$ in comparison with AA although higher than standardized PBE. Therefore, CPE has low activity to inhibit the elastase enzyme which is responsible for degradation of elastin fibrous structure in a dermal matrix.

CPE exhibited almost two times better collagenase inhibition activity than AA and three times better activity than standardized PBE based on $\mathrm{IC}_{50}$ (Table 5). Increasing the concentration of the tested sample to more than $1000 \mu \mathrm{g} / \mathrm{ml}$ (data not shown) did not affect the collagenase inhibition activity, as exhibited by standardized PBE. Research by Lim et al. [68] indicated that kaempferol and quercetin were strong inhibitors of collagenase while apigenin, a flavone compound, weakly inhibited collagenase activity. Flavonols had better inhibition activity towards collagenase enzyme than flavones compounds [69]. Resveratrol, a stilbenoid compound, was patented for its effective inhibition against the collagenase effectively in reducing wrinkles [70]. CPE showed significantly higher collagenase inhibition activity than standardized PBE at $1000 \mu \mathrm{g} / \mathrm{ml}$, although the activity was lower than that of AA and control inhibitor. The higher inhibition by CPE can be attributed to the presence of more than one flavonol compound, whereas PBE has only one identified flavonol compound. In addition, CPE was detected with two types of resveratrol compared with $\mathrm{PBE}$. Therefore, the presence of various polyphenolic compounds in CPE contributed to its potential in maintaining collagen longevity in the skin layer [4].

\section{Skin whitening and UV-protecting potential}

The capability of CPE to inhibit tyrosinase activity can be translated to its potential as skin whitening agent. When tyrosinase enzyme activity is inhibited, melanin production is reduced, resulting in a fairer skin. Inhibition of tyrosinase activity was significantly higher in $\mathrm{CPE}$ than standardized $\mathrm{PBE}$ at high concentration $(1000 \mu \mathrm{g} / \mathrm{ml})$ but showed similar activity at lower concentration $(125 \mu \mathrm{g} / \mathrm{ml})$. CPE was also found to be a better inhibitor of tyrosinase activity than kojic acid and ascorbic acid (Table 6), although not as good as PBE based on the $\mathrm{IC}_{50}$ value. $\mathrm{CPE}$ and PBE contained several compounds, such as flavonols, flavanols, stilbenoids and phenolic acid, reported to inhibit tyrosinase activity [6]. Quercetin, a flavonol compound, inhibits tyrosinase enzyme better than kaempferol [71] suggesting higher inhibition activity by PBE [58] than CPE. Resveratrol in CPE contributed to the inhibition of the enzyme; however, this contribution was less than that of oxyresveratrol [72]. Fatty acids, such as linoleic acid and oleic acid, which were detected in the CPE too, 
Table 8 Cell viability (\%) of the CPE against HDFa in comparison with PBE and AA

\begin{tabular}{|c|c|c|c|c|c|c|c|c|c|c|}
\hline \multirow[t]{2}{*}{ Sample } & \multicolumn{9}{|c|}{ Concentration, $\mu \mathrm{g} / \mathrm{ml}$} & \multirow[t]{2}{*}{$I_{90}$} \\
\hline & 3.9 & 7.8 & 15.6 & 31.3 & 62.5 & 125 & 250 & 500 & 1000 & \\
\hline AA & $14.35 \pm 3.04^{b a}$ & $18.70 \pm 1.84^{c a}$ & $22.17 \pm 0.61^{c \beta}$ & $36.96 \pm 4.30^{\text {ay }}$ & $40.87 \pm 3.69^{\complement \delta}$ & $108.26 \pm 24.90^{\mathrm{a} \varepsilon}$ & $590.43 \pm 19.68^{\mathrm{a} \epsilon}$ & $1407.39 \pm 46.12^{\mathrm{a} f}$ & $651.30 \pm 133.04^{\mathrm{a} \epsilon}$ & 57.10 \\
\hline PBE & $17.95 \pm 1.21^{b a}$ & $24.79 \pm 2.42^{\mathrm{b} \beta}$ & $29.49 \pm 1.81^{\text {by }}$ & $42.31 \pm 5.44^{\mathrm{a} \delta}$ & $69.23 \pm 1.21^{\text {a }}$ & $118.35 \pm 1.81^{\mathrm{a} \epsilon}$ & $202.14 \pm 42.91^{\mathrm{bE}}$ & $335.47 \pm 17.53^{\mathrm{b \zeta}}$ & $285.47 \pm 49.56^{\mathrm{b} £ \zeta}$ & 101.21 \\
\hline CPE & $368.47 \pm 46.15^{\mathrm{a \gamma}}$ & $62.32 \pm 4.49^{\mathrm{a} \beta}$ & $40.80 \pm 7.41^{\mathrm{aa}}$ & $39.92 \pm 2.74^{\mathrm{aa}}$ & $55.62 \pm 8.32^{\mathrm{b} \beta}$ & $58.47 \pm 6.26^{\mathrm{b} \beta}$ & $61.75 \pm 11.20^{c \beta}$ & $76.83 \pm 12.25^{c \beta}$ & $62.82 \pm 10.11^{c \beta}$ & 7.42 \\
\hline
\end{tabular}

a b c d Different alphabet in column means significantly different within concentration.

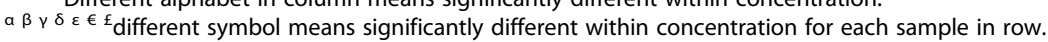


were also reported to have skin whitening properties without adverse effects [73].

Absorbance at UVC spectrum $(200-290 \mathrm{~nm})$ is not discussed as the radiation is filtered by the atmosphere. Meanwhile, absorbance of the tested samples at the other two spectrums; i.e., UVB (290-315 nm) and UVA $(315-400 \mathrm{~nm})$, are discussed. There are two ways in which sunscreen agent in cosmetic products protect the skin: (i) by scattering the UV-rays (titanium and zinc oxide) or (ii) by absorbing the light before reaching the skin. Plant extract, especially with flavones [74] and pigments, can absorb UV light [67]. CPE showed potentially good UV absorbance at UVB range wavelength, which was even better than the commercially used UV-protecting agent in this study; i.e. Avobenzone and Octylmethoxycinnamate (OMC), and significantly better than standardized PBE at $290 \mathrm{~nm}$ (Table 7). Higher sun-protecting action in CPE could be caused by flavone derivatives such as luteolin [75] at UVA wavelength $(330 \mathrm{~nm})$, which were absent in PBE. The presence of methyl salicylate compound in CPE also enhanced the absorbance [76]. CPE also showed similar absorbance potential to that of commercial UV-sunscreen agent, OMC, at $308 \mathrm{~nm}$, the peak of erythema (sunburn) action spectrum [77]. The extract had significantly better absorbance than Avobenzone at this wavelength.

CPE had moderate absorbance of UVA (315-400 nm) as illustrated in Figure 1, which was significantly better than that of standardized PBE (Table 7). Therefore, CPE has better potential as UVB sunscreen agent for cosmetic formulation and can be recommended in combination with other sunscreen agents with higher UVA absorbance properties. All plant extracts with pigment and color can absorb UVA and or UVB [67] as CPE does. Extracts with color might not be possible for white cream formulation; however, for color cosmetic products; i.e. foundation and lipstick, colored extracts could be added as sunscreen agents.

\section{Cell viability using human dermal fibroblasts}

In this study we calculated the $\mathrm{IC}_{90}$, the concentration of the tested solution to maintain the cell population up to $90 \%$. High value of $\mathrm{IC}_{90}$ indicated that high concentration of the tested solution is needed to maintain the population of fibroblast cell. Table 8 shows that CPE had the lowest value indicating low concentration of the extract was able to maintain the cell growth at $90 \%$. The highest value obtained by $\mathrm{PBE}$, which was almost two times more than that of AA. Almost no toxicity was exhibited by CPE at the tested concentration since the cell viability percentage was more than $50 \%$, in comparison with AA and PBE. There could be possible cell death at low concentrations for $\mathrm{AA}$ at $62.5 \mu \mathrm{g} / \mathrm{ml}$ and $\mathrm{PBE}$ at
$31.3 \mu \mathrm{g} / \mathrm{ml}$, in contrast to the results of a study conducted by Kim et al. [58] in which PBE showed no toxicity to B16 melanoma cell, a type of cell different from the one used in this study. At high concentrations of AA and $\mathrm{PBE}$, the cells proliferated as shown by the increasing percentage of more than $100 \%$, the highest percentage being achieved at $500 \mu \mathrm{g} / \mathrm{ml}$. Contrary to CPE, at low concentration of the tested solution $(3.9 \mu \mathrm{g} / \mathrm{ml})$, the percentage of cell viability was the highest, thus low concentration of $\mathrm{CPE}$ was needed to induce proliferation of fibroblast cell.

\section{Conclusions}

We concluded that cocoa pod extract exhibits better antioxidant activities potential than standardized pine bark extract as measured using DPPH scavenging and $\beta$ carotene bleaching assays, but lower activities using FRAP assay, based on $\mathrm{EC}_{50}$. CPE inhibited collagenase and elastase enzymes better than PBE, although second to PBE in inhibiting the tyrosinase enzyme. Cocoa pod extract also possesses high potential as UVB sunscreen agent although with lower performance in UVA range wavelength than that of the commercial sunscreen agent. However, the extract can be formulated with other UVA protecting agents to work synergistically for a broad spectrum of UV exposure. We, therefore, recommend a possible use of CPE as ingredient for functional cosmetic products specifically for anti-wrinkles as well as skin whitening or sunscreen products in combination with natural plant extracts to widen the spectrum of protecting from sun-rays.

\section{Abbreviations}

AA: Ascorbic acid; BCB: $\beta$-carotene bleaching; BHT: Butylated hydroxytoluene; CPE: Cocoa pod extract; DMEM: Dulbecco's modified Eagle's medium; DMSO: Dimethyl sulfoxide; DNTB: Dinitrothiocyano benzene; DPPH: 2-diphenyl2-picrylhydrazyl; EC 5 : Effective concentration at 50\%; ECM: Extracellular matrix; FBS: Fetal bovine serum; FRAP: Ferric Reducing Antioxidant Power; GAE: Gallic Acid Equivalent; GS1: Nebulising gas; GS2: Heating gas; HDFa: Human dermal fibroblast adult; HEPES: Hydroxyethyl piperazineethanesulfonic acid; $I_{50}$ : Inhibition concentration at 50\%; I $C_{90}$ : Inhibition concentration at 90\%; KA: Kojic acid; LC-MS/MS: Liquid chromatography mass spectrometry/mass spectrometry; L-DOPA: L-3,4-dihydroxyphenyl-alanine methyl ester hydrochloride; MMP-1: Matrix metalloproteinase-1; MS IDA EPI: Mass spectrometry information dependant acquisition enhanced product ion; MTT: 3-(4,5-Dimethylthiazol-2-yl)-2,5-diphenyl tetrazolium bromide; NNGH: N-Isobutyl-N-(4-methoxyphenylsulfonyl) glycylhydroxamic acid; OMC: Octylmethoxycinnamate; PBE: Pine bark extract; PBS: Phosphate buffer saline; ROS: Reactive oxygen species; TFC: Total flavonoid content; TPC: Total phenolic content; TPTZ: 2,4,6-tripyridyl-s-triazine; UHPLC: Ultra high performance liquid chromatography; UV: Ultraviolet.

Competing interests

The authors declare that they have no competing interests.

\section{Authors' contributions}

AAK conceived of the study, participated in its experimentation and coordination, performed the statistical analysis and drafted the manuscript. AA involved in the design of the study and helped to draft the manuscript. Al designed the study and supervised the experimental workflow. PH drafted the experimental flow and helped to draft the manuscript. SSAG involved in 
data arrangement and help to draft the manuscript. BHZ carried out the LCMS/MS experimental work, performed the LCMS analysis and drafted this section. NAA performed the antioxidant assay and helped to draft the manuscript. All authors read and approved the final manuscript.

\section{Acknowledgements}

The authors would like to thank Analytical Service Laboratory of Malaysian Cocoa Board for using laboratory facilities. Special gratitude extended to Malaysian Cocoa Board for funding the research. Authors would like to acknowledge Universiti Putra Malaysia for consultation and expertise advice to complete the research work

\section{Author details}

${ }^{1}$ Cocoa Innovation and Technology Centre, Malaysian Cocoa Board, PT12621, Nilai Industrial Area, 71800 Nilai, Negeri Sembilan, Malaysia. ${ }^{2}$ Department of Nutrition and Dietetics, Faculty of Medicine and Health Sciences, Universiti Putra Malaysia, 43400 UPM Serdang, Selangor, Malaysia. ${ }^{3}$ Laboratory of Halal Science Research, Halal Products Research Institute, Universiti Putra Malaysia, 43400 UPM Serdang, Selangor, Malaysia. ${ }^{4}$ Research Centre of Excellence, Nutrition and Non-communicable Disease, Faculty of Medicine and Health Sciences, Universiti Putra Malaysia, 43400 UPM Serdang, Selangor, Malaysia.

Received: 17 February 2014 Accepted: 1 October 2014

Published: 7 October 2014

\section{References}

1. Mukherjee PK, Maity N, Nema NK, Sarkar BK: Bioactive compounds from natural resources against skin aging. Phytomedicine 2011, 19(1):64-73.

2. Kim YH, Chung CB, Kim JG, Ko Kl, Park SH, Kim JH, Eom SY, Kim YS, Hwang $\mathrm{Yl}$, Kim KH: Anti-wrinkle activity of Ziyuglycoside I isolated from a Sanguisorba officinalis root extract and its application as a cosmeceutical ingredient. Biosci Biotechnol Biochem 2008, 72(2):303-311.

3. Purba MB, Kouris-Blazos A, Wattanapenpaiboon N, Lukito W, Rottenberg EM, Steen BC, Wahlqvist Ml: Skin wrinkling: can food make a difference? J Am Coll Nutr 2001, 20(1):71-80.

4. Landau M: Exogenous factors in skin aging. In Environmental Factors in Skin Diseases. Curr Probl Dermatol. Edited by Tur E. Basel: Karger; 2007:1-13.

5. Svobodova A, Walterova D, Vostalova J: Ultraviolet light induced alteration to the skin. Biomed Pap Med Fac Univ Palacky Olomouc Czech Repub 2006, 150(1):25-38.

6. Lin JW, Chiang HM, Lin YC, Wen KC: Natural products with skin-whitening effects. J Food Drug Anal 2008, 16(2):1-10

7. Masaki H: Role of antioxidants in the skin: Anti-aging effects. J Derm S 2010, 58:85-90.

8. Pouillot A, Polla LL, Tacchini P, Neequaye A, Polla A, Polla B: Natural antioxidants and their effects on the skin. In Formulating, Packaging, and Marketing of Natural Cosmetic Products. Edited by Dayan N, Kromidas L. New Jersey: John Wiley \& Sons Inc; 2011.

9. Figueira A, Janick J, BeMiller JN: New products from Theobroma cacao: Seed pulp and pod gum. In New Crops. Edited by Janick J, Simon JE. New York: Wiley; 1993:475-478 [online]

10. Samuel YKC: Crude gum from cocoa of Malaysian origin: Part 1 : Rheological Properties. Malaysian Cocoa Journal 2006, 2:28-31.

11. Sartini M, Gemini A: Effect of cocoa pod husk extracts on prevention of hypercholesterolemia in albino rats (Rattus novergicus). Majalah Farmasi \& Teknologi 2008, 12(3):78-82.

12. Vriesmann LC, de Mello Castanho Amboni RD, de Oliveira Petkowicz CL: Cacao pod husks (Theobroma cacao L.): composition and hot-watersoluble pectins. Ind Crops Prod 2011, 34:1173-1181.

13. Padh H: Cellular functions of ascorbic acid. Biochem Cell Biol 1990, 68(10):1166-1173.

14. Murad S, Tajima S, Johnson GR, Sivarajah A, Pinnell SH: Collagen synthesis in cultured human skin fibroblasts: Effect of ascorbic acid and its analogs. J Invest Dermatol 1983, 81:158-162

15. Patil $Y$, Soman $G$, Shiney $P$, Wagle A: Evaluation of in vitro antioxidant activity of herbage of aromatic plants. J Cell Tissue Res 2010, 10(1):2125-2129.

16. Kim JH, Byun JC, Bandi AKR, Hyun CG, Lee NM: Compounds with elastase inhibition and free radical scavenging activities from Callistemon lanceolatus. J Med Plants Res 2009, 3(11):914-920.
17. Benzie IFF, Strain JJ: The ferric reducing ability of plasma (FRAP) as a measure of "antioxidant power": The FRAP assay. Anal Biochem 1996, 239:70-76.

18. Szollosi $R$, Varga IS: Total antioxidant power in some species of Labiatae (Adaption of FRAP method). Acta Biologica Szegediensis 2002, 46(3-4):125-127. In Proceedings of the $7^{\text {th }}$ Hungarian Congress on Plant Physiology, S2-P24.

19. Katalinic V, Milos M, Kulisic T, Jukic M: Screening of 70 medicinal plant extracts for antioxidant capacity and total phenols. Food Chem 2006, 94:550-557.

20. Arnous A, Makris DP, Kefalas P: Correlation of pigment and flavonoid content with antioxidant properties in selected aged regional wines from Greek. J Food Comp Anal 2002, 15:655-665.

21. Pulido R, Bravo L, Calixto FS: Antioxidant activity of dietary polyphenols as determined by a modified ferric reducing/antioxidant power assay. J Agric Food Chem 2000, 48:3396-3402.

22. Bub A, Watzl B, Abrahamse L, Delince' e H, Adam S, Wever J, Mu“ller H, Rechkemmer G: Moderate intervention with carotenoid-rich vegetable products reduces lipid peroxidation in men. J Nutr 2000, 130(9):2200-2206.

23. Othman A, Ismail A, Ghani NA, Adenan I: Antioxidant capacity and phenolic content of cocoa beans. Food Chem 2007, 100:1523-1530.

24. Marxen K, Vanselow KH, Lippemeier S, Hintze R, Ruser A, Hansen UP: Determination of DPPH radical oxidation caused by methanolic extracts of some microalgae species by linear regression analysis of spectrophotometric measurements. Sensor 2007, 7(10):2080-2095.

25. Shahidi F, Naczk M: Chapter 8: Antioxidant properties of food phenolic. In Phenolics in Food and Nutraceuticals 2004:403-442.

26. Kulisic T, Radonic A, Katalinic V, Milos M: Use of different methods for antioxidative activity of oregano essential oil. Food Chem 2004, 85:633-640.

27. Enzo Life S: Matrix Metalloproteinase-1 (MMP-1) colorimetric drug discovery kit. http://www.enzolifesciences.com/BML-AK404/matrixmetalloproteinase-1mmp-1-colorimetric-drug-discovery-kit (accessed on 28 December 2012).

28. Enzo Life Sciences: Neutrophil elastase colorimetric drug discovery kit. http:// www.enzolifesciences.com/BML-AK497/neutrophil-elastasecolorimetric-drugdiscovery-kit (accessed on 22 February 2013).

29. Chiari M, Joray MB, Ruiz G, Palacios SM, Carpinella MC: Tyrosinase inhibitory activity of native plants from central Argentina: Isolation of an active principle from Lithraea molleoides. Food Chem 2010, 120(1):10-14.

30. Bobin MF, Raymond M, Martini MC: UVA/UVB absorption properties of natural products. Cosmet Toiletries 1994, 109:63-70.

31. Joe MJ, Kim SN, Choi HY, Shin WS, Park GM, Kang DW, Kim YK: The inhibitory of Eckol and Dieckol from Ecklonia stolonifera on the expression of matrix metalloproteinase-1 in human dermal fibroblasts. Biol Pharm Bull 2006, 29(8):1735-1739.

32. Chueahongthong F, Ampasavate C, Okonogi S, Tima S, Anuchapreeda S: Cytotoxic effects of crude kaffir lime (Citrus hystrix, DC.) leaf fractional extracts on leukemic cell lines. J Med Plants Res 2011, 5(14):3097-3105.

33. Weyermann J, Lochmann D, Zimmer A: A practical note on the use of cytotoxicity assays. Int J Pharm 2005, 288:369-376.

34. High Quality Mass Spectral Database. In http://www.massbank.jp. Date access: 12/12/13

35. ReSpect for Phytochemicals. In http://spectra.psc.riken.jp. Date access: 12/12/13.

36. Schäfer $A$, Högger $P$ : Oligomeric procyanidins of French maritime pine bark extract $\left(\right.$ Pycnogenol ${ }^{\oplus}$ ) effectively inhibit a-glucosidase. Diabet Res Clin Pr 2007, 77:41-46.

37. Sun J, Liang F, Bin Y, Li P, Duan C: Screening non-colored phenolics in red wines using liquid chromatograpy/ultraviolet and mass spectrometry/ mass spectrometry libraries. Molecules 2007, 12:679-693.

38. Rabaneda FS, Jauregui O, Casals I, Lacueva CA, Pulido MZ, Raventos ML: Liquid chromatographic/electrospray ionization tandem mass spectrometric study of the phenolic composition of cocoa (Theobroma cacao). J Mass Spectrom 2003, 38:35-42.

39. Reidah IMA, Roman DA, Carretero AS, Gutierrez AF: Profiling of phenolic and other polar constituents from hydro-methanolic extract of watermelon (Citrullus lanatus) by means of accurate-mass spectrometry (HPLC-ESI-QTOF-MS). Food Res Int 2013, 51(1):354-362.

40. Santos SAO, Pinto PCRO, Silvestre AJD, Neto CP: Chemical composition and antioxidant activity of phenolic extracts of cork from Quercus suber L. Ind Crops Prod 2010, 31(3):521-526. 
41. Pierson JT, Monteith GR, Thomson SJR, Dietzgen RG, Gidley MJ, Shaw PN: Phytochemical extraction, characterization and comparative distribution across four mango (Mangifera indica L.) fruit varieties. Food Chem 2014, 149(15):253-263.

42. Sawada Y, Akiyama K, Sakata A, Kuwahara A, Otsuki H, Sakurai T, Saito K, Hirai MY: Widely targeted metabolomics based on large-scale MS/MS data for elucidating metabolite accumulation patterns in plants. Plant Cell Physiol 2009, 50:37-47. doi:10.1093/pcp/pcn183.

43. Dias MI, Barros L, Duenas M, Pereira E, Carvalho AM, Alves RC, Oliveira BPP, Buelga CS, Ferreira ICFR: Chemical composition of wild and commercial Achillea millefolium L. and bioactivity of the methanolic extract, infusion and decoction. Food Chem 2013, 141(4):4152-4160.

44. Sawada Y, Nakabayashi R, Yamada Y, Suzuki M, Sato M, Sakata A, Akiyama K, Sakurai T, Matsuda F, Aoki T, Hirai MY, Saito K: RIKEN tandem mass spectral database (ReSpect) for phytochemicals: A plant-specific MS/MS-based data resource and database. Phytochem 2012, 82:38-45.

45. Souza LM, Cipriani TR, lacomini M, Gorin PAJ, Sassaki GL: HPLC/ESI-MS and NMP analysis of flavonoids and tannins in bioactive extract from leaves of Maytenus ilicifolia. J Pharm Biomed Anal 2008, 47(1):59-67.

46. Jin A, Ozga JA, Lutz DL, Schieber A, Reinecke DM: Characterization of proanthocyanidins in pea (Pisum sativum L.), lentil (Lens cullinaris L.) and faba bean (Vicia faba L.) seeds. Food Res Int 2012, 46(2):528-535.

47. Romero MG, Carretero AS, Gutierrez AF: Metabolite profiling and quantification of phenolic compound in methanol extracts of tomato fruit. Phytochem 2010, 71(16):1848-1864.

48. Medina ICR, Carretero AS, Gutierrez AF: Use of high-performance liquid chromatography with diode array detection coupled to electrospray-Qqtime of flight mass spectrometry for the direct characterization of the phenolic fraction in organic commercial juices. J Chrom A 2009, 1216(23):4736-4744

49. Roesler R, Catharino RR, Malta LG, Eberlin MN, Pastore G: Antioxidant activity of Annona crassiflora: Characterization of major components by electrospray ionization mass spectrometry. Food Chem 2007, 104(3):1048-1054.

50. Bystrom LM, Lewis BA, Brown DL, Rodriguez E, Obendorf RL Characterisation of phenolics by LC-UV/Vis, LC-MS/MS and sugars by GC in Melicoccus bijugatus Jacq'.Montgomery' fruits. Food Chem 2008, 111(4):1017-1024.

51. Pereira LB, Angulo I, Losada PP, Cruz JM: Phenolic profile and antioxidant properties of crude extract obtained from a brewery waste stream. Food Res Int 2013, 51(2):663-669.

52. Deng J, Fan C, Yang Y: Identification and determination of the major constituents in Deng's herbal tea granules by rapid resolution liquid chromatography coupled with mass spectrometry. J Pharm Biomed Anal 2011, 56(5):928-936.

53. Harada K, Ohyama Y, Tabushi T, Kobayashi A, Fukusaki E: Quantitative analysis of anionic metabolites for Catharanthus roseus by capillary electrophoresis using sulfonated capillary coupled with electrospray ionization-tandem mass spectrometry. J Biosci Bioeng 2008, 105(3):249-260

54. Herrero M, Plaza M, Cifuentes A, Ibanez E: Green processes for extraction of bioactives from rosemary: Chemical and functional characterization via UPLC-MS/MS and in-vitro assays. J Chrom A 2010, 1217(16):2512-2520.

55. Lou Z, Wang H, Zhu S, Zhang M, Gao Y, Ma C, Wang Z: Improved extraction and identification by ultra performance liquid chromatography tandem mass spectrometry of phenolic compounds in burdock leaves. J Chrom A 2010, 1217(16):2441-2446.

56. Barros L, Duenas M, Carvalho AM, Ferreira ISFR, Buelga CS: Characterization of phenolic compounds in flowers of wild medicinal plants from North eastern Portugal. Food Chem Toxicol 2012, 50:1576-1582.

57. Carazzone C, Mascherpa D, Gazzani G, Papetti A: Identification of phenolic constituents in red chicory salads (Cichorium intybus) by highperformance liquid chromatography with diode array detection and electrospray ionisation tandem mass spectrometry. Food Chem 2013, 138(2-3):1062-1071.

58. Kim YJ, Kang KS, Yokozawa T: The anti-melanogenic effect of pycnogenol by its anti-oxidative actions. Food Chem Toxicol 2008, 46:2466-2471.

59. Mishra K, Ojha $\mathrm{H}$, Chaudhury NK: Estimation of antiradical properties of antioxidants using DPPH • assay: A critical review and results. Food Chem 2011, 130(4):1036-1043.

60. Psotova J, Lasovsky J, Vicar J: Metal-chelating properties, electrochemical behavior, scavenging and cytoprotective activities of six natural phenolics. Biomed Papers 2003, 147(2):147-153.
61. Rodrigues E, Poerner N, Rockenbach II, Gonzaga LV, Mendes CR, Fett R: Phenolic compounds and antioxidant activity of blueberry cultivars grown in Brazil. Cienc Tecnol Aliment Capinas 2011, 31(4):911-917.

62. Graßmann J: Terpenoids as plant antioxidants. Vitam Horm 2005, 7:505-535.

63. Fremont L, Belguendouz L, Delpal S: Antioxidant activity of resveratrol and alcohol-free wine polyphenols related to LDL oxidation and polyunsaturated fatty acids. Life Sci 1999, 64(21):2511-2521.

64. Grimm T, Schäfer A, Högger P: Antioxidant activity and inhibition of matrix metalloproteinases by metabolites of maritime pine bark extract (Pycnogenol). Free Radical Biol Med 2004, 36(6):811-822.

65. Thring TSA, Hili P, Naughton DP: Anti-collagenase, anti-elastase and anti-oxidant activities of extracts from 21 plants. BMC Comp Altern Med 2009, 9(27). doi:10.1186/1472-6882-9-27.

66. Sahasrabudhe A, Deodhar M: Anti-hyaluronidase, anti-elastase activity of Garcinia indica. Int J Bot 2010, 6(3):299-303.

67. Nichols JA, Katiyar SK: Skin photoprotection by natural polyphenols: anti-inflammatory, anti-oxidant and DNA repair mechanisms. Arch Dermatol Res 2010, 302(2):71-86.

68. Lim H, Kim HP: Inhibition of mammalian collagenase, matrix metalloproteinase-1, by naturally-occurring flavonoids. Planta Med 2007 73(12):1267-1274.

69. Sin BY, Kim HP: Inhibition of collagenase by naturally-occurring flavonoids. Arch Pharm Res 2005, 28(10):1152-1155.

70. Breton L, Livier C, Fagot D: Skin toning by stimulating collagen synthesis/ proliferation of dermal fibroblasts. In U.S. Patent No. 6,147,121; 2000.

71. Chen QX, Kubo I: Kinetics of mushroom tyrosinase inhibition by quercetin. J Agric Food Chem 2002, 50:4108-4112.

72. Ohguchi K, Tanaka K, llyya I, Ito T, linuma M, Matsumoto K, Asao Y, Nozawa Y: Gnetol as a potent tyrosinase inhibitor from genus Gnetum. Biosci Biotechnol Biochem 2003, 67:663-665.

73. Parvez S, Kang M, Chung HW, Cho C, Hong MC, Shin MK, Bae H: Survey and mechanism of skin depigmenting and lightening agents. Phytother Res 2006, 20:921-934.

74. Elumalai A, Eswariah MC, Didla A, Kasarla R, Narumalla N: A preliminary study on in-vitro evaluation of flowers of Sesbania grandiflora (Linn) as a natural anti-solar agent. Int J of Phytopharmacol 2012, 3(1):18-20.

75. Fischer F, Zufferey E, Bourgeois JM, Heritier J, Micaux F: UV-ABC screens of luteolin derivatives compared to edelweiss extract. J Photochem Photobiol B Biol 2011, 103:8-15.

76. Ramu G, Kumar GS, Ramesh B: Anti-solar activities of Lantana camara L. plants in yellow and red flowers. Res J Pharm Biol Chem Sci 2012, 3(3):981-986.

77. Rutter K, Sell DR, Fraser N, Obrenovich M, Zito M, Starke-Reed P, Monnie VM: Green tea extract suppress the age-related increase in collagen cross-linking and fluorescent products in C57BL/6 mice. Int J Vitam Nutr Res 2003, 73(6):453-460.

doi:10.1186/1472-6882-14-381

Cite this article as: Abdul Karim et al:: Phenolic composition, antioxidant, anti-wrinkles and tyrosinase inhibitory activities of cocoa pod extract. BMC Complementary and Alternative Medicine 2014 14:381.

\section{Submit your next manuscript to BioMed Central and take full advantage of:}

- Convenient online submission

- Thorough peer review

- No space constraints or color figure charges

- Immediate publication on acceptance

- Inclusion in PubMed, CAS, Scopus and Google Scholar

- Research which is freely available for redistribution 